\title{
Diagnostic Accuracy of Contrast Enhanced Computed Tomography in Detection of Ovarian Cancer in Clinically Suspected Patients
}

\author{
Gulzar Bhund, Abid Ali Sahito, Mohsin Hussain Khoso, Sasui Memon, Hafeez ur Rehman, Ahsan Ullah
}

\begin{abstract}
Background: In the gynecologic malignancies, ovarian cancer is the $2^{\text {nd }}$ most common and a big cause of the mortality. Contrast-enhanced CT is a recent imaging technique of choice in preoperative assessment of ovarian cancer. Objective: To determine the diagnostic accuracy of contrast enhanced computed tomography of abdomen and pelvis in detection of ovarian cancer in clinically suspected patients by using histopathology as gold standard. Study Design: Cross-Sectional Study. Settings: Department of Radiology Civil Hospital, Karachi Pakistan. Duration: Six months from $26^{\text {th }}$ April to 25th October 2017. Methodology: All the clinically suspected patients of ovarian cancer were included. Contrast Enhanced Computed Tomography (CECT) of pelvis and abdomen was performed with injection of intravenous contrast material. The CECT, diagnostic accuracy was established in terms of sensitivity, specificity, PPV, NPV against histopathology. By taking p-value $\leq 0.05$ as significant, chi square test (post stratification) was applied. Results: The mean age of patients was $31.84 \pm 7.95$ years. Mean duration of symptoms was $13.37 \pm 5.99$ weeks. Serum cancer antigen-125 level was $62.23 \pm 14.66 \mathrm{U} / \mathrm{ml}$. Total $27.5 \%$ subjects were diagnosed with ovarian cancer by contrast enhanced CT and $29.6 \%$ by Histopathology. Specificity, Sensitivity, NPV, PPV, and accuracy were $86.7 \%, 97.4 \%, 93.4 \%, 94.5 \%$, and $94.2 \%$ respectively. Conclusion: The contrast enhanced computer tomography is helpful diagnostic tool to detect the ovarian cancer, with accuracy rate of $94.2 \%, 86.7 \%$ sensitivity and $97.4 \%$ specificity.
\end{abstract}

Keywords: Diagnostic Accuracy, Contrast Enhanced Computed Tomography, Ovarian Cancer.

Corresponding Author

Submitted for Publication: 05-08-2019

Accepted for Publication: 21-10-2019

DR. GULZAR BHUND, Consultant Radiologist, National Medical Center Hospital, Karachi-Pakistan

Contact / Email: +92 333-2700119, aabid_sahito@hotmail.com

Citation: Bhund G, Sahito AA, Khoso MH, Memon S, Rehman H, Ullah A. Diagnostic Accuracy of Contrast Enhanced Computed Tomography in

Detection of Ovarian Cancer in Clinically Suspected Patients. APMC 2020;14(1):66-9.

\section{INTRODUCTION}

The ovarian cancer has become one of the commonest causes of death in gynecological malignancies and the 4th most common causative factor of death in women from cancers within the United States and European nation. In Pakistan, Cancer data from institutional study shows that ovarian cancer accounts for $13.6 \%$ of cancers in females. ${ }^{1}$ Moreover, it remains the 2 nd commonest causative factor of death among women from malignancies following breast tumor. ${ }^{2}$

The cause of ovarian cancers includes several risk factors. Late menopause is also associated with an increased risk. ${ }^{3}$ Prevalence rates in developing countries are the largest, with rates in those regions approaching 9 per 0.1 million (excluding Japan where incidence rate is 64 per 1 million). Prevalence rates in several western nations and Japan have been rising gradually. Oral contraceptives usage and high parity minimize the ovarian cancer risk. In India, as per numerous hospitalbased cancer databases, the age-adjusted prevalence of ovarian cancer varies between 50 and 83 cases per 0.1 million females. The median age in India is 45 years at the time of diagnosis, which is around 10 years lower than in developed nations. ${ }^{4} \mathrm{CT}$ is also the first method used for detecting ovarian cancer. Since ovarian cancer symptoms suggest advanced disease yet are usually non-specific (such as, palpable abdominal weight, distention or abdominal, early satiety, urinary frequency), CT is achieved for ascites or occult intra-abdominal malignancy. ${ }^{5}$ Serum cancer antigen -125 (CA-125) is a tumor indicator for ovarian carcinoma and when it is elevated more than normal value of $35 \mathrm{U} / \mathrm{ml}$, it has been observed to be associated with ovarian malignancy in $80 \%$ of women however with early disease in only $50 \%$ of females. ${ }^{6,7}$ Ultrasound remains the first line intervention for determining adnexal pathologies, but mostly it cannot differentiate benign lesions from malignant ones and the severity of disease in cancerous cases therefore further evaluation is done using serum CA-125 levels, CT scan and nuclear MRI and in certain cases laparoscopy. ${ }^{8}$ Computed Topography is the preferred investigation in planning additional administration among patients with metastatic disease as well as it also enables to comprehensively evaluate the primary tumor and the site of lymphadenopathy and peritoneal metastasis so the ovarian masses can be distinguished and features concerning the malignancy and benignity can be observed ${ }^{8}$. One study performed in Pakistan ${ }^{9}$ showed specificity and sensitivity of CT scan in detection of ovarian masses as 86.7 and $92 \%$ respectively, while another study from India ${ }^{10}$ showed specificity of CT scan in detection of ovarian malignancy as $46.2 \%$. Petru et al 11 studied 175 patients suspected of having an ovarian mass found $70 \%$ sensitivity. Contrast enhanced computed tomography is commonly available noninvasive and useful diagnostic investigation to detect ovarian cancer. Various studies in literature showed marked differences ${ }^{9-11}$ in results of sensitivity and specificity of CT scan in the detection of ovarian cancer. These variations give strong rationale to conduct this study in our population to detect diagnostic accuracy of CT scan in clinically suspected ovarian cancer patients. 


\section{METHODOLOGY}

Study Design: Cross-Sectional Study.

Settings: This study was conducted in Radiology Department of Civil Hospital, Karachi Pakistan.

Duration: Six months from 26 ${ }^{\text {th }}$ April 2017 to $25^{\text {th }}$ October 2017. Sample Size: By taking sensitivity $=92 \%$, specificity $=86.7 \%$, Prevalence $=13.6 \%{ }^{1}$, margin of error $=8 \%$, and $95 \%$ confidence level. The calculated sample size was 331 patients

Inclusion Criteria: Age 31 - 70 years. Clinically suspected ovarian cancer as ultrasound features and elevated serum CA125 levels with presence of vague pelvic pain of mild intensity that do not prohibit patient from performing routine work, feeling of pelvic swelling or heaviness, urinary urgency and frequency and palpable pelvic mass on clinical examination

Exclusion Criteria: Biopsy proven cases of ovarian cancer. History of previous surgery involving either ovary. Serum creatinine greater than $1.5 \mathrm{mg} / \mathrm{dl}$ or with history of chronic renal failure. Pregnant women.

Methods: Study was done on patients those were referred to the Radiology department of Civil Hospital Karachi for CT scan abdomen ted after approval from CPSP. Purpose and procedure of study were explained including the risks and benefits. After taking an informed consent, CECT of abdomen and pelvis performed at Radiology department Civil Hospital Karachi by senior radiologist with experience of at least 5 years on Toshiba Activion Multislice Computed Tomography scanner with injection of intravenous contrast material. Computed Tomography was performed in the axial plane with multi-planner reformations in sagittal and coronal planes with the patient lying in supine position. Ovarian cancer was diagnosed on the basis of appearances as

cystic or complex ovarian mass of $>4 \mathrm{~cm}$ in diameter with enhancing thick irregular walls $(>3 \mathrm{~mm})$ and multiple thick septations in cystic ovarian masses, pelvic or/and abdominal lymphadenopathy with loss of fatty hilum and architectural destruction and hepatic metastatic deposits appearing hypodense or/and Peritoneal metastatic deposits appearing isoto hyperdense. Findings of CT scan were recorded on research proforma and histopathology results reported by consultant pathologist at Dow University of Health Science Laboratory Karachi were then collected and recorded on proforma. Data was analyzed by SPSS version 20 . Mean \pm SD were calculated for numerical data and frequency and percentages were calculated for categorical data. A 2x2 table was constructed and sensitivity, specificity, PPV, NPV and accuracy of CT of abdomen and pelvis for detection of ovarian cancer was estimated by using histopathology as gold standard.

\section{RESULTS}

Total 331 patients were selected and the mean age of the respondents was $55.68 \pm 8.80$ years. The mean duration of symptoms of study participants was $13.37 \pm 5.99$ weeks and the mean serum CA-125 level was $62.23 \pm 14.66 \mathrm{U} / \mathrm{ml}$. $23.9 \%$ subjects were pre-menopausal and rests of the $76.1 \%$ were post-menopausal. Unilateral side was involved in $41.7 \%$ subjects and bilateral side was involved in $58.3 \%$ subjects as presented in Table 1.

Table 1: Basic statistical data of patients $(n=331)$

\begin{tabular}{|c|c|c|c|}
\hline \multicolumn{2}{|c|}{ Variables } & Frequency & $\%$ \\
\hline \multirow{2}{*}{ Side Involved } & Unilateral & 138 & 41.7 \\
\hline & Bilateral & 193 & 58.3 \\
\hline \multirow{2}{*}{ Menopausal Status } & Pre-Menopausal & 79 & 23.9 \\
\hline & Post-Menopausal & 252 & 76.1 \\
\hline \multirow{2}{*}{ Age Groups } & $\leq 55$ years & 159 & $48 \%$ \\
\hline & $>55$ years & 172 & $52 \%$ \\
\hline \multirow{2}{*}{ Gravidity } & $\leq 3$ & 238 & $72 \%$ \\
\hline & $>3$ & 93 & $28 \%$ \\
\hline \multirow{2}{*}{ Parity } & $\leq 3$ & 222 & $67 \%$ \\
\hline & $>3$ & 109 & $33 \%$ \\
\hline Age (mean+SD) & \multicolumn{3}{|c|}{$55.68 \pm 8.80$ years } \\
\hline $\begin{array}{c}\text { Symptoms duration } \\
\text { (mean+SD) }\end{array}$ & \multicolumn{3}{|c|}{$13.37 \pm 5.99$ weeks } \\
\hline CA-125 (mean+SD) & \multicolumn{3}{|c|}{$62.23 \pm 14.66 \mathrm{U} / \mathrm{ml}$} \\
\hline
\end{tabular}

$27.5 \%$ were diagnosed with ovarian cancer by contrast enhanced computed tomography. As far as Histopathology findings are concerned, ovarian cancer was diagnosed in $29.6 \%$ study subjects as presented in Table 2.

Sensitivity, Specificity, Predictive values and diagnostic accuracy of contrast enhanced computed tomography for the detection of ovarian cancer taking histopathology as gold standard were calculated. The results showed that 85 patients were true positive, correctly diagnosed and 227 patients were true negative, correctly diagnosed. Sensitivity, Specificity, PPV, NPV and accuracy were $86.7 \%, 97.4 \%, 93.4 \%, 94.5 \%$, and $94.2 \%$ respectively as presented in Table 2 .

Table 2: Diagnostic accuracy of computed tomography (CT) by taken histopathology as gold standard $(n=331)$

\begin{tabular}{|c|c|c|c|c|}
\hline & \multicolumn{3}{|c|}{ Histopathology } & \multirow{2}{*}{ P-value } \\
\cline { 1 - 4 } CT & Yes & No & TOTAL & \multirow{2}{*}{0} \\
\cline { 1 - 4 } Present & 85 & 6 & 91 & \multirow{2}{*}{$0.000^{*}$} \\
\cline { 1 - 4 } Absent & 13 & 227 & 240 & \\
\cline { 1 - 3 } TOTAL & 98 & 233 & 331 & \multirow{2}{*}{ Accuracy } \\
\hline Sensitivity & Specificity & PPV & NPV & 94.2\% \\
\hline 86.7\% & $97.4 \%$ & $93.4 \%$ & $94.5 \%$ & \\
\hline
\end{tabular}




\section{DISCUSSION}

Ovarian cancer remains the most common causative factor of death from gynecological malignancies and is the 4th most common causative factor of cancer-related death among females in the United States and Europe. ${ }^{12}$ Cancer data revealed from an institutional study across Pakistan; indicate that female breast carcinoma was the most prevalent cancer with $38.5 \%$ of malignancies among female followed by ovarian carcinoma (13.6\%).12 CT understates the staging and pelvic assessment of pelvic masses by a gynecologist and serum CA125 along with its sensitivity is frequently below $50 \%$ in diagnosis of pelvic masses. ${ }^{13}$ In this study the sensitivity, specificity, predictive values and diagnostic accuracy of contrast enhanced computed tomography for the detection of ovarian cancer taking histopathology as gold standard were calculated. The results showed that 85 patients were true positive, correctly diagnosed and 227 patients were true negative, correctly diagnosed. Sensitivity, Specificity, PPV, NPV and accuracy were $86.7 \%, 97.4 \%, 93.4 \%, 94.5 \%$, and $94.2 \%$ respectively. The sensitivity of morphological examination with ultrasound to predict malignancy in ovarian cancers has been reported to be around $85 \%-97 \%$, while its precision varies from $56 \%$ $95 \% .{ }^{13-15}$ Kinkel et al, in their meta-analysis reported that CT reveals specificity and sensitivity of $87 \%$ and $81 \%$ respectively, for indeterminate masses observed on ultrasound..$^{16}$ Likewise, Liu et al revealed that CT/PET scanner exhibits a $87 \%$ sensitivity and $100 \%$ specificity in distinguishing benign and malignant ovarian malignancies. ${ }^{17}$ Tsili et al as well reported that adnexal masses can be categorized by MDCT into malignant and benign in around $89 \%$ and $93 \%$ of cases. ${ }^{18}$ One study also reported $97 \%$ sensitivity and $91 \%$ specificity. Ovarian mass appearance on CT varies greatly, so it is not necessarily possible to provide precise histological characterization. For each tumor type, certain radiological findings prevail; understanding of these main characteristics of ovarian malignancies enable for a particular diagnosis or significant filtering of differential diagnosis. ${ }^{19,20}$ The presence of ovarian cancers in the image varies between cystic through solid mass. While cancers have identical radiological and clinical findings, every form of ovarian cancer has predominant or different main features. ${ }^{21-23}$ However, the recent developments in CT technologies have allowed better detection and enhanced function, not just in differentiating benign ovarian masses from malignant masses, however also in assessing disease extent and metastatic deposits. Adequate estimation of the type of mass and severity of the disease remains valuable in treatment intervention that saves the patients from needless surgical procedure and $\operatorname{cost}^{18,20}$

\section{LIMITATIONS}

According to the limitations of present study, it was done on the small scale and at the urban environment therefore, findings might not be generalizable to larger populations.

\section{CONCLUSION}

It was concluded that contrast enhanced computed tomography is the satisfied diagnostic tool, with accuracy rate of $94.2 \%$ showing $86.7 \%$ sensitivity and $97.4 \%$ specificity and it could be used for detection of ovarian cancer.

\section{SUGGESTIONS / RECOMMENDATIONS}

Because ovarian cancer has been documented to be the prominent trigger of death among gynecological cancers and that there is inadequate data on the epidemiology of epithelial ovarian ovarian cancer in country, a large population-based research is needed in Pakistan to pave the way for ovarian cancer control and prevention in the area.

\section{CONFLICT OF INTEREST / DISCLOSURE} None.

\section{ACKNOWLEDGEMENTS}

I am very thankful to Department of Radiology of Civil Hospital, Karachi Pakistan to provide technical support for completion of this research work.

\section{REFERENCES}

1. Iqbal R, Hussain ARK, Intsar A. One-year review of cases of ovarian malignancy at fatima memorial hospital. Pak J Med Health Sci. 2013;7(4):1134-6.

2. Saler, E, Eliyahaus S, Leleg D, Tsabari A. Laparoscopic management of adenexal cystic mass in post-memopausal women. Obstet Gynecol. 1994;83(3):594-6.

3. Muhabat $Q$, Waheed $F$, Waqarunissa, Jabeen N. Clinical presentation of ovarian tumors. Open J Obstet Gynecol. 2016;6205-9.

4. Chandrashekhara SH, Thulkar S, Srivastava DN, Kumar L, Hariprasad R, Sharma MC, et al. Pre-operative evaluation of peritoneal deposits using multidetector computed tomography in ovarian cancer. Brit J Radiol. 2011;84(997):38-43.

5. Jeong YY, Outwater EK, Kang HK. Imaging evaluation of ovarian masses. Radio Graphics. 2000;20(5):1445-70.

6. Kauff ND, Satagopan JM, Robson ME, Offit K. Risk-reducing salpingo-oophorectomy in women with a BRCA1 or BRCA2 mutation. N Engl J Med. 2002;346(21):1609-15.

7. Gupta D, Lis CG. Role of CA125 in predicting ovarian cancer survival - a review of the epidemiological literature. J Ovarian Res. 2009:2-13.

8. Mubarak F, Alam MS, Akhtar W, Hafeez S, Nizamuddin N. Role of multidetector computed tomography (MDCT) in patients with ovarian masses. Int J Wom Health. 2011;3:123-6.

9. Khattak YJ, Hafeez S, Alam T, Beg M, Awais M, Masroor I. Ovarian masses: is multi-detector computed tomographya reliable imaging modality? Asian Pacific J Cancer Prev. 2013;14(4):2627-30.

10. Moideen N, Habbar SS, Rai L, Guruvare S, Adiga P. Comparison of CA-125, conventional ultrasound and CT imaging in diagnosis and staging of ovarian cancer correlated with surgicopathological finding. Int J Reprod Contracept Obstet Gynecol. 2014;3(4):924-30.

11. Petru E, Schmidt F, Mikosch P, Pickel H, Lahousen $M$, Tammussino $\mathrm{K}$ et al. Abdominopelvic computed tomography in 
the preoperative evaluation of suspected ovarian masses. Int $\mathrm{J}$ Gynecol Cancer. 1992;2(5):252-5.

12. Aziz Z, Sana S, Saeed S, Akram M. Institution based tumor registry from Punjab: five-year data-based analysis. J Pak Med Assoc. 2003;53(8):350-3.

13. Tempany CM, Zou KH, Silverman SG, Brown DL, Kurtz AB. Staging of advanced ovarian cancer: comparison of imaging modalities-report from the Radiological Diagnostic Oncology Group. Radiology. 2000;215(3):761-7.

14. Baert AL, Forstner R. Encyclopedia of diagnostic imaging: carcinoma ovarium. Vol 1. New York: Springer; 2008:259.

15. Nam E, Kim Y, Kim J. Kim. Diagnosis and staging of ovarian cancer: comparative values of PET/CT, Doppler US, CT, and MRI correlated with histopathologic analysis. J Clin Oncol. 2008;26(15): 5567.

16. Kinkel K, Lu Y, Mehdizade A, Pelte MF, Hricak H. Indeterminate ovarian mass at ultrasound: incremental value of second imaging test for characterization-meta analysis and Bayesian analysis. Radiology. 2005;236(1):85-94.
17. Liu Y. Benign ovarian and endometrial uptake on FDG PET-CT: patternsand pitfalls. Ann Nucl Med. 2009;23(2):107-112.

18. Tsili AC, Tsampoulas C, Charisiadi A. Adnexal masses: accuracy of detection and differentiation with multidetector computed tomography. Gynecol Oncol. 2008;110(1):22-31.

19. Imaoka I, Wada A, Kaji Y. Developing an MR imaging strategy for diagnosis of ovarian masses. Radiographics. 2006;26(5):143148.

20. Tamai K, Koyama T, Saga T. MR features of physiologic and benign conditions of the ovary. Eur Radiol. 2006;16(12):2700-11.

21. Brown DL, Zou KH, Tempany CMC. Primary versus secondary ovarian malignancy: imaging findings of adnexal masses in the Radiology Diagnostic Oncology Group Study. Radiology. 2001;219(1):213-8.

22. Andersen ES, Knudsen A, Rix P, Johansen B. Risk of malignancy index in the preoperative evaluation of patients with adnexal masses. Gynecol Oncol. 2003;90(1):109-12.

23. Altekruse SF, Kosary CL, Krapcho M. SEER Cancer Statistics Review, 1975-2007, National Cancer Institute. Bethesda, MD. http://seer.cancer.gov/csr/1975_2007/.

\section{AUTHORSHIP AND CONTRIBUTION DECLARATION}

\begin{tabular}{lc} 
AUTHORS & Contribution to The Paper \\
\hline $\begin{array}{l}\text { Dr. Gulzar Bhund } \\
\text { Consultant Radiologist, } \\
\text { NMC Hospital, Karachi Pakistan }\end{array}$ & Data collect and manuscript writing \\
\hline $\begin{array}{l}\text { Dr. Abid Ali Sahito } \\
\text { Assistant Professor, Radiology } \\
\text { Bilawal Medical College, LUMHS Jamshoro Pakistan }\end{array}$ & Research idea, Manuscript writing \& Data analysis \\
\hline $\begin{array}{l}\text { Dr. Mohsin Hussain Khoso } \\
\text { Senior Registrar, Pir Abdul Qadir Shah Jilani }\end{array}$ \\
$\begin{array}{l}\text { Institute of Medical Science Gambat Khairpur } \\
\text { Pakistan }\end{array}$ \\
$\begin{array}{l}\text { Dr. Sasui Memon } \\
\text { Consultant Radiologist, } \\
\text { Advance Diagnostic Center, } \\
\text { Bilawal Medical College, LUMHS Jamshoro Pakistan }\end{array}$ \\
$\begin{array}{l}\text { Dr. Hafeez ur Rehman } \\
\text { Senior Registrar, Indus Medical College Hospital } \\
\text { Tando Muhammad Khan Pakistan }\end{array}$ \\
$\begin{array}{l}\text { Dr. Ahsan-Ullah } \\
\text { Consultant Radiologist } \\
\text { Matiari Health Services, Matiari Pakistan }\end{array}$
\end{tabular}

